\title{
Cefaleia do esforço, displasia fibromuscular do tronco braquiocefálico e hipoplasia das artérias carótidas comum, interna e externa à direita: um estudo de caso
}

\author{
Effort headache, fibromuscular dysplasia of the brachiocephalic trunk and hypoplasia of \\ the common carotid arteries, internal and external to the right: a case study
}
Cefalea de esfuerzo, displasia fibromuscular del tronco braquiocefálico e hipoplasia de las arterias carótidas comunes, interna y externa a la derecha: estúdio de un caso

Talita Silva Alves $^{1 *}$, Tamyris Silva Alves ${ }^{1}$, Ricardo Soffiatti Mesquita de Oliveira ${ }^{2}$, Lineu Domingos Miziara ${ }^{1}$.

\section{RESUMO}

Objetivo: Relatar um caso original de cefaleia do esforço (CE), displasia fibromuscular (DFM), hipoplasia de carótidas e síndrome do roubo da subclávia em adulto jovem. Detalhamento de Caso: Em 2010, paciente, 26 anos, masculino, branco, passou a apresentar cefaleia, bifrontoparietal, de forte intensidade, latejante, preferindo deitar-se, acompanhada por foto e fonofobia, às vezes náuseas, com duração de 4 a 24 horas, que só acontecia algumas horas após o término de exercícios físicos, mas nunca durante os mesmos. Em 2017, o paciente apresentava exame físico normal, exceto por pulso radial diminuído à direita e pulso carotídeo abolido à direita. No doppler de carótidas e vertebrais, observaram-se alterações sugerindo roubo parcial da artéria subclávia direita. A arteriografia carotídea e vertebral bilateral revelou estenose irregular de $80 \%$ no terço proximal do tronco braquiocefálico, sugestiva de DFM, e, logo após, estenose de $60 \%$, que se prolonga até as artérias carótidas comum, interna e externa à direita, com inversão do fluxo em artéria vertebral direita e tronco tireocervical. Ademais, evidenciou provável hipoplasia de artérias carótidas comum, interna e externa à direita. Considerações finais: Foi relatado um caso original, que merece atenção, pois não se pode dar certeza se a CE está relacionada às alterações vasculares.

Palavras-chave: Cefaleia, Síndrome do roubo subclávio, Displasia fibromuscular, Hipoplasia.

\begin{abstract}
Objective: To report an original case of exertional headache (EH), fibromuscular dysplasia (FDM), carotid hypoplasia and subclavian theft syndrome in a young adult. Case Detail: In 2010, a 26-year-old male white patient began to suffer a severe, bilateral, frontoparietal, throbbing headache. During the attacks, he prefered to lie down, had photo and phonophobia and sometimes nausea. The attacks lasted 4 to 24 hours and happened only a few hours after the end of physical exercises, but never during them. In 2017, the patient had a normal physical examination, except for a decreased radial pulse on the right and an abolished carotid pulse on the right. The carotid and vertebral dupplex showed alterations suggesting partial theft of the right subclavian artery. Bilateral carotid and vertebral arteriography revealed irregular $80 \%$ stenosis in the proximal third of the brachiocephalic trunk, suggestive of FDM, and $60 \%$ stenosis extending to the common carotid arteries, right internal and external carotid arteries, with inversion of the flow in the right vertebral artery and thyreocervical trunk. Moreover, probable hypoplasia of common carotid arteries, right internal and external carotid artery was revealed. Final considerations: An original case was reported, which deserves attention, because it cannot be certain whether the EC is related to the vascular changes.
\end{abstract}

Key words: Headache, Subclavian steal syndrome, Fibromuscular dysplasia, Hypoplasia.

1 Universidade de Uberaba (UNIUBE), Uberaba - MG. *E-mail: talita_alves03@edu.uniube.br

2 Universidade Federal do Triângulo Mineiro, Uberaba - MG.

SUBMETIDO EM: 1/2021

ACEITO EM: 2/2021

PUBLICADO EM: 2/2021 


\section{RESUMEN}

Objetivo: Informar sobre un caso original de cefalea de esfuerzo (EC), displasia fibromuscular (DFM), hipoplasia carotídea y síndrome de robo de la subclavia en un adulto joven. Detalle del caso: En 2010, un paciente masculino, blanco de 26 años comenzó a presentar dolor de cabeza, bifrontoparietal, de fuerte intensidad, punzante, prefiriendo acostarse, acompañado de foto y fonofobia, a veces con náuseas, que duró de 4 a 24 horas, lo que solo ocurrió unas horas después de terminar los ejercicios físicos, pero nunca durante ellos. En 2017, el paciente tuvo un examen físico normal, excepto por la disminución del pulso radial en la derecha y la abolición del pulso carotídeo en la derecha. Se observaron alteraciones en el dúplex carotídeo y vertebral que sugieren un robo parcial de la arteria subclavia derecha. La arteriografía carotídea y vertebral bilateral reveló una estenosis irregular del $80 \%$ en el tercio proximal del tronco braquiocefálico, sugerente de DFM, y poco después, una estenosis del $60 \%$ que se extiende a las arterias carótidas comunes, internas y externas a la derecha, con inversión del flujo en la arteria vertebral derecha y el tronco tirocervical. Además, reveló una probable hipoplasia de las arterias carótidas comunes, internas y externas a la derecha. Consideraciones finales: Se comunicó un caso original, que merece atención, porque no se puede asegurar que el CE esté relacionado con alteraciones vasculares.

Palabras clave: Dolor de cabeza, Síndrome del robo de la subclavia, Displasia fibromuscular, Hipoplasia.

\section{INTRODUÇÃO}

A cefaleia do esforço (CE) primária é reconhecida desde 1988 pela International Headache Society como um possível diagnóstico de dor de cabeça. Essa patologia é benigna, autolimitada, de curta duração e é desencadeada pelo exercício físico. Geralmente, $50 \%$ dos pacientes com esse tipo de cefaleia podem ter histórico de migrânea. De acordo com alguns estudos, estima-se que a cefaleia do esforço afeta de 1 a $26 \%$ dos adultos (SANDOE C, et al., 2018).

A síndrome do roubo da subclávia é uma patologia vascular rara em que há uma inversão do fluxo de sangue da artéria vertebral ipsilateral, devido à oclusão da artéria subclávia, ou mesmo do tronco braquiocefálico, o que é mais raro. É uma doença mais prevalente no sexo masculino, em torno dos 60 anos de idade. O diagnóstico se baseia no Doppler pulsado que analisa a artéria vertebral e identifica a reversão do fluxo sanguíneo. Para os pacientes sintomáticos o tratamento é cirúrgico. Em estudos mais recentes, a angioplastia transluminal percutânea é uma alternativa razoável para o tratamento (PASSOS MD, et al., 2016).

Já na hipoplasia da artéria carótida interna $(\mathrm{ACl})$, que é uma anomalia congênita rara, ocorre um desenvolvimento incompleto dessa artéria, o que ocasiona um estreitamento do seu calibre. Não encontramos, na literatura, casos de hipoplasia da artéria carótida externa (ACE) e comum (ACC) (CALDAS MA, et al., 2019).

Por fim, de acordo com Persu A, et al. (2014), a displasia fibromuscular (DFM) é definida como uma doença idiopática segmentar, não aterosclerótica e não inflamatória da musculatura das paredes arteriais, levando à estenose das artérias de pequeno e médio calibre. Essa doença acomete principalmente as mulheres, com diagnóstico entre 50 e 55 anos, conforme padrões angiográficos e histopatológicos (DI MONACO S, et al., 2014).

Assim, o presente estudo teve como objetivo relatar um caso de um paciente com displasia fibromuscular, hipoplasia de carótidas, síndrome do roubo da subclávia e cefaleia do esforço.

\section{DETALHAMENTO DE CASO}

Paciente de 26 anos, masculino, branco, procurou atendimento médico com história de cefaleia bifrontoparietal há 7 anos, que se iniciava com leve intensidade e se tornava de forte intensidade em aproximadamente 1 hora. Essa cefaleia era latejante, cursava com foto e fonofobia, às vezes náuseas. Durante a mesma o paciente preferia deitar-se. A dor durava de 4 a 24 horas, não piorava com os esforços de rotina, mas a dor acontecia horas após o término do exercício físico, nunca durante os mesmos.

Em 2014, o paciente foi submetido a uma angiorressonância magnética arterial encefálica, que revelou assimetria dos calibres das $\mathrm{ACl}$ (menor à direita), discretas irregularidades dos contornos do segmento 
petroso da $\mathrm{ACl}$ direita, artéria cerebral posterior esquerda originando-se da $\mathrm{ACl}$ ipsilateral, assimetria dos fluxos do segmento V4, aumento do calibre da artéria comunicante posterior direita, imagem alveolar com vazio de fluxo localizada em topografia paravertebral da região cervical superior direita (podendo representar estrutura venosa).

Além disso, foi solicitada uma angiografia venosa por ressonância, observando-se uma assimetria dos seios transversos. Foi realizado o diagnóstico de migrânea sem aura, e prescrito propranolol $40 \mathrm{mg}$ duas vezes ao dia. Em 2017, o paciente, devido à persistência da cefaleia, procurou outro neurologista. No exame físico, foram observados diminuição do pulso radial à direita e pulso carotídeo abolido à direita. O propranolol foi mantido, e foram acrescentados amitriptilina $25 \mathrm{mg}$ ao dia e clorpromazina $7 \mathrm{mg}$ ao dia. Foram solicitados dupplex de artérias carótidas e vertebrais e doppler arterial e venoso de membros superiores.

No doppler de carótidas e vertebrais, observou-se inversão parcial da onda sistólica em todo o sistema carotídeo vertebral direito, redução do calibre dos vasos carotídeos à direita, e os vasos carotídeos e a artéria vertebral esquerda estavam com fluxo anterógrado preservado, espessura das camadas mediointimais das carótidas comuns normal, velocidade dos fluxos do lado esquerdo normal e velocidade não detectada do lado direito, demonstrando sinais de roubo parcial da artéria subclávia direita. $O$ doppler venoso de membros superiores não apresentou alterações. Todavia, o doppler arterial de membros superiores apresentou sinais indiretos de estenose significativa da artéria braquiocefálica.

O paciente, então, foi submetido a uma arteriografia carotídea e vertebral bilateral (março de 2018), que demonstrou aorta torácica de calibre normal, tronco braquiocefálico com estenose irregular de $80 \%$ no terço proximal em formato de "colar de contas", sugestivo de DFM, além de estenose de $60 \%$, irregular, difusa, prolongando-se até as $\mathrm{ACC}, \mathrm{ACl}$ e $\mathrm{ACE}$ à direita, com inversão do fluxo em artéria vertebral direita e tronco tireocervical (Figura 1).

Figura 1 - Arteriografia com alteração sugestiva de "colar de contas" e hipoplasia ACC à direita.

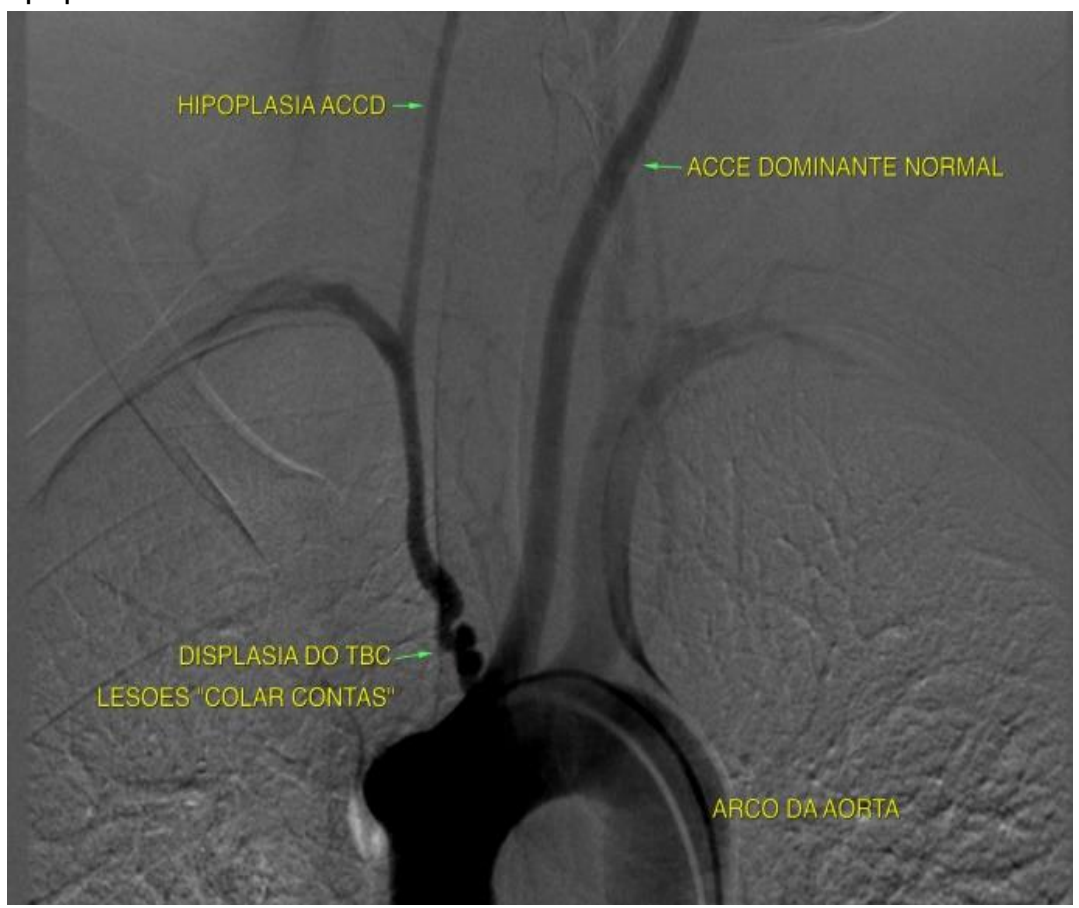

Fonte: Alves TS, et al., 2021.

A ACC direita estava hipoplásica, com fluxo de fino calibre, de $5 \mathrm{~mm}$ em toda extensão, e a $\mathrm{ACl}$ e $\mathrm{ACE}$ revelaram-se com fino calibre, também em toda a extensão (Figura 2). Ademais, não se detectaram sinais de aneurismas ou malformações e a parte venosa e o lado esquerdo estavam normais. 
Figura 2 - Arteriografia com hipoplasia de $\mathrm{ACC}$ e $\mathrm{ACl}$ à direita.

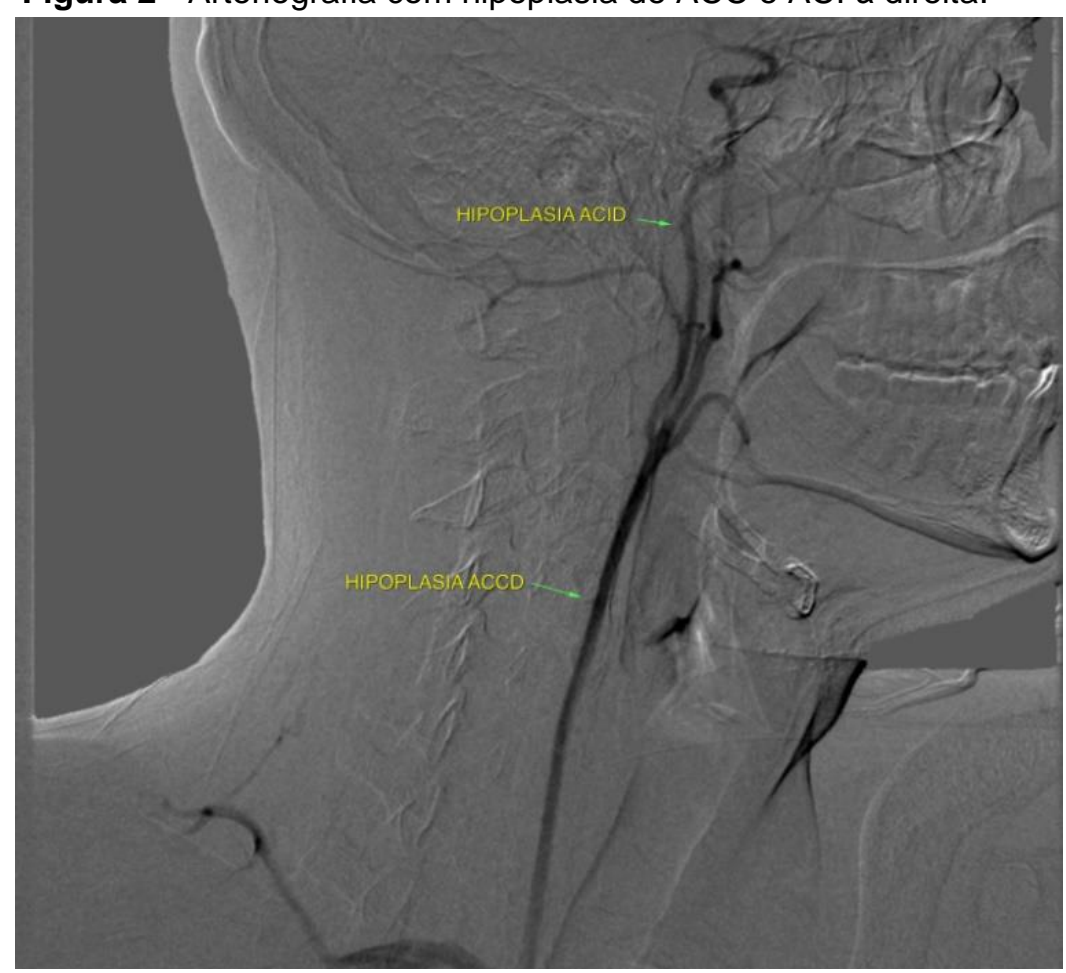

Fonte: Alves TS, et al., 2021.

Foram estabelecidos, assim, os diagnósticos de migrânea sem aura, sem relação com o quadro de DFM, e hipoplasia do sistema carotídeo direito associado à DFM do tronco braquiocefálico, com hipoplasia das ACC, ACI e ACE direitas. Devido ao bom fluxo colateral, e ao fato da DFM e as hipoplasias serem assintomáticas, foi adotado tratamento conservador. Além disso, foram prescritos nortriptilina $50 \mathrm{mg}$ ao dia e ácido acetilsalić́lico $100 \mathrm{mg}$ ao dia. O paciente foi orientado a não realizar esportes coletivos, musculação e a evitar medicamentos vasoconstrictores.

\section{DISCUSSÃO}

A CE, segundo a International Headache Society (2018), é uma cefaleia primária, mais comum no sexo masculino. Geralmente, trata-se de um quadro benigno, autolimitado e idiopático. No entanto, devido ao fato de ser potencialmente secundária a alguma alteração, como tumor de fossa posterior e hemorragia subaracnóidea, um primeiro episódio de CE requer avaliação precoce e minuciosa, a fim de se estabelecer o diagnóstico e iniciar o tratamento (UPADHYAYA P, et al., 2020).

A CE geralmente acontece após exercícios físicos extenuantes e a maioria dos pacientes apresenta cefaleia que se inicia cerca de 30 a 60 minutos após o exercício. Essa dor usualmente é bilateral, pulsátil ou compressiva, com duração de 5 minutos a 48 horas (TURNER J, 2003).

A fisiopatologia da CE primária não é bem estabelecida, mas acredita-se que esteja relacionada a alterações vasculares, como a vasodilatação venosa e arterial decorrente do exercício, ocasionando a dor. Além disso, estudos demonstram que a congestão venosa intracraniana oriunda do fluxo venoso jugular retrógrado, decorrente da incompetência da válvula da veia jugular interna poderia explicar o quadro por levar a um aumento da pressão intracraniana. Essas alterações, contudo, não foram detectadas no caso relatado (SANDOE C, et al., 2018).

O diagnóstico da CE é realizado a partir dos critérios da International Classification of Headache Disorders (ICHD-3), descritos no Quadro 1. Há necessidade de investigação através de exames complementares, como ressonância magnética de crânio. No caso descrito, o paciente preenchia os critérios da ICHD-3 para CE e 
realizou os exames de imagem para investigação de doenças secundárias. Além disso, é importante lembrar que alguns casos de CE estão relacionados a doença arterial coronariana (raramente, a dor da isquemia miocárdica pode se irradiar para a cabeça). Finalmente, devemos avaliar se a cefaleia não preenche os critérios para migrânea desencadeada pelo esforço, pois, nesse caso, fica estabelecido o diagnóstico de migrânea, ao invés de CE (SANDOE C, et al., 2018).

Quadro 1 - Critérios de diagnóstico da ICHD - 3 para cefaleia primária por exercício.

\begin{tabular}{|l|}
\hline Cefaleia primária por exercício \\
\hline A: Pelo menos dois episódios de dor de cabeça que preenchem os critérios B e C \\
\hline B: Ocasionada apenas durante ou após exercícios físicos extenuantes \\
\hline C: Duração < 48h \\
\hline D: Não é explicada por outro diagnóstico na ICHD-3 \\
\hline Provável cefaleia primária por exercício \\
\hline A: Pode ser por \\
\hline 1: um único episódio de cefaleia que preenche critérios B e C \\
\hline 2: pelo menos dois episódios de cefaleia que preencham o critério B, mas não o C \\
\hline B: Ocasionada apenas durante ou após exercícios físicos extenuantes \\
\hline C: Duração < 48h \\
\hline D: Não atende os critérios ICHD-3 para qualquer outro distúrbio de cefaleia \\
\hline E: Não é explicada por outro diagnóstico na ICHD-3 \\
\hline
\end{tabular}

Fonte: Alves TS, et al., 2021.

O tratamento da CE não está bem definido. Especialistas orientam um aquecimento mais demorado antes da atividade física, aumentar gradualmente a intensidade do exercício, ou que se deixe de praticar a atividade que costuma desencadear a dor. No caso descrito, o paciente foi orientado a evitar determinados tipos de esporte. Recomenda-se o uso de indometacina ou betabloqueadores, como propranolol, e para a prevenção de ataques de cefaleia podem ser usados o tartarato de ergotamina ou flunarizina (QUEIROZ LP, 2004).

A síndrome do roubo da subclávia, de acordo com Passos MD, et al. (2016), constitui uma patologia vascular, com inversão do fluxo sanguíneo da artéria vertebral ipsilateral, devido a uma estenose mais distal dessa mesma artéria, oclusão da artéria subclávia, ou, mais raramente, do tronco braquiocefálico. A arteriosclerose é a principal causa de oclusão da artéria subclávia, principalmente, na porção proximal da subclávia esquerda. A proporção de lesões sintomáticas na artéria subclávia esquerda, em relação à direita, é de 3:1 (ZIMMERMAN NB, 1993).

Essa síndrome ocorre em virtude de uma obstrução que provoca redução da pressão na artéria subclávia. O sangue flui pela artéria vertebral contralateral, atinge a artéria basilar e desce mais lentamente pela artéria vertebral ipsilateral, fornecendo sangue para o membro superior por uma circulação colateral. No exame físico do nosso paciente, foi observada diminuição do pulso radial direito e abolição de pulso carotídeo ipsilateral (BEER A, 2012).

A síndrome do roubo da subclávia é uma doença rara, que acomete principalmente o sexo masculino, em torno dos 60 anos. É assintomática em $6 \%$ dos pacientes com sopros cervicais. Os sintomas mais frequentes são síncope, vertigem e claudicação intermitente da extremidade superior ipsilateral (LOPES MNSC, et al., 2013).

O diagnóstico, segundo Passos MD, et al. (2016), é feito com doppler pulsado, que consegue identificar o fluxo sanguíneo reverso na artéria vertebral. Com isso, possibilita-se a classificação da síndrome, conforme 
as alterações hemodinâmicas, em: tipo 1 ou latente, quando as alterações são mínimas, tipo 2 ou intermitente/parcial quando são moderadas e tipo 3 ou completo, se as alterações forem acentuadas. 0 tratamento visa reverter de forma definitiva o fluxo anterógrado na artéria vertebral acometida, reduzindo a hipoperfusão cerebral e os sintomas, sendo realizado através da cirurgia ou da angioplastia transluminal percutânea em pacientes sintomáticos (FREGNI F, et al., 2003).

Já na hipoplasia da $\mathrm{ACl}$, ocorre desenvolvimento incompleto e estreitamento do calibre arterial, o qual pode ser visualizado 1 a $2 \mathrm{~cm}$ acima da bifurcação carotídea e pode continuar ao longo do trajeto intracraniano. De acordo com Caldas MA, et al. (2019), é uma anomalia rara, que acomete menos de $0,01 \%$ da população quando bilateral. Quando bilateral, a compensação do suprimento sanguíneo ocorre pelo sistema vertebrobasilar; quando unilateral, a compensação se dá pela carótida contralateral dominante. Além disso, a hipoplasia da ACl, geralmente, não apresenta manifestações clinicas (SÁNCHEZ-MONTAÑO M, et al., 2016).

Atualmente, não há diretrizes específicas para o controle da hipoplasia. Entretanto, devido ao alto risco de aneurisma e acometimento cerebral, torna-se necessário monitoramento da pressão arterial, recomendandose alterações dos hábitos de vida e, em alguns casos, uso de anti-hipertensivos (HOU D, et al., 2019).

A DFM, de acordo com Prisant LM, et al. (2006), consiste em uma patologia vascular não inflamatória e não aterosclerótica frequentemente, encontrada no sexo feminino, em caucasianos e na faixa de 50 a 55 anos. É um problema que acomete artérias de pequeno e médio calibre. A classificação é feita a partir da camada acometida, sendo a DFM de camada média a mais comum (PERES LAB, et al., 2007).

A etiologia não é muito bem estabelecida, mas Pontes TC, et al. (2012) acredita que esteja relacionado a uma herança autossômica dominante e ao estrogênio, o que explicaria a predominância em mulheres. Outras hipóteses etiológicas são descritas, como início precoce de tabagismo, isquemia intramural, entre outras (DI MONACO S, et al., 2018).

De acordo com Van de Nes JA, et al. (2007) as artérias renais e cérvico-cervicais são as mais acometidas (60-75\% e $25-30 \%$, respectivamente), podendo apresentar a doença simultaneamente. Todavia, vasos viscerais, coronarianos e ilíacos também são afetados. As manifestações clínicas ocorrem de acordo com o leito arterial acometido e pelo grau de comprometimento do fluxo sanguíneo, por exemplo, hipertensão arterial secundária quando a alteração está nas artérias renais, sintomas cerebrovasculares se em artérias carótidas, isquemia mesentérica quando em vasos viscerais. O paciente também pode estar assintomático com alterações eletrocardiográficas, se as coronárias estiverem lesadas (PONTES TC, et al., 2012).

O acometimento de artérias cérvico-cervicais pode acarretar, segundo Pontes TC, et al. (2012), além de sintomas inespecíficos, como cefaleia, vertigem e zumbido, complicações, incluindo aneurismas, acidente vascular encefálico e ataque isquêmico transitório. Na maioria dos estudos, as carótidas são afetadas. Porém, no caso descrito, foi evidenciado acometimento do tronco braquiocefálico, com estenose irregular de $80 \%$ no terço proximal, em formato sugestivo de DFM.

O diagnóstico de DFM, conforme Prisant LM, et al. (2006) baseia-se nos padrões angiográficos e histopatológicos, importantes também para analisar qual camada está sendo afetada. Segundo Pontes TC, et al. (2012), quando a camada média está lesada, o padrão visualizado é o "colar de contas"; quando a camada intima é a afetada, o padrão será de estenose focal e concêntrica; e quando a camada adventícia for acometida o padrão será de estenose tubular. O tratamento da displasia ainda está em discussão, mas, em casos sintomáticos, realiza-se a revascularização cirúrgica ou por angioplastia transluminal percutânea (PERES LAB, et al., 2007).

Portanto, descrevemos o caso de um paciente com CE, e as seguintes alterações vasculares: síndrome do roubo da subclávia, DFM do tronco braquiocefálico e hipoplasia das ACC, ACI e ACE à direita. Não encontramos, na literatura, relatos associando CE com as demais patologias, assim não podemos dizer com certeza se essas patologias foram achadas fortuitas, ou se estão relacionadas à fisiopatologia da cefaleia em questão. Logo, trata-se de uma condição que merece relevância, visto que as alterações vasculares podem ocasionar novos sintomas ao paciente. 


\section{REFERÊNCIAS}

1. BEER A. Síndrome do roubo da subclávia. Revista da Sociedade Brasileira de Angiologia e de Cirurgia Vascular, 2012; 3: 13-18.

2. CALDAS MA, et al. Hipoplasia Bilateral de Carótida Interna em Paciente Assintomática. Relato de Caso. Arq Bras Cardiol: Imagem cardiovascular, 2019; 32(4):331-333.

3. DI MONACO S, et al. Genomics of Fibromuscular Dysplasia. Int J Mol Sci., 2018; 19(5): 1526.

4. FREGNI F, et al. Treatment of subclavian steal syndrome with percutaneous transluminal angioplasty and stenting: case report. Arq Neuropsiquiatr., 2003; 61(1): 95-9.

5. HOU D, et al. Hipoplasia congênita da artéria carótida interna: relato de caso. Medicina (Baltimore), 2019; 98(1).

6. INTERNATIONAL HEADACHE SOCIETY. The International Classification of Headache Disorders,3rd edition. Cephalalgia: sage journals, 2018; 38(1): 1-211.

7. VAN DE NES JA, et al. Fibromuscular dysplasia of the basilar artery: an unusual case with medico-legal implications. Forensic Sci Int, 2007; 173(2-3): 188-192.

8. LOPES MNSC, et al. Síndrome do roubo subclávio: relato de caso. Brasília Med. 2013; 50(2): 2013.

9. OLESEN J, et al. The Headaches, Raven Press, 1993.

10. PASSOS MD, et al. Atualização sobre Ultrassom Doppler das Artérias Vertebrais: Síndrome do Roubo da Subclávia. Arq Bras Cardiol: Imagem cardiovascular, 2016; 29(2): 58-62.

11. PERES LAB, et al.Displasia Fibromuscular. Relato de Caso. Jornal Brasileiro de Nefrologia, 2007; 29(3).

12. PERSU A, et al. European consensus on the diagnosis and management of fibromuscular dysplasia. J. Hypertens, 2014; 32: 1367-1378.

13. PONTES TC, et al. Displasia fibromuscular: um diagnóstico diferencial para as vasculites. Rev. Bras. Reumatol., $2012 ; 52(1)$.

14. PRISANT LM, et al. Fibromuscular dysplasia: an uncommon cause of secondary hypertension. J Clin Hypertens, 2006; 8(12): 894-8.

15. QUEIROZ LP. Cefaleias associadas ao esforço físico e à atividade sexual. Headache Medicine. 2004; 7(3): $115-119$.

16. SÁNCHEZ-MONTAÑO M, et al. Hipoplasia bilateral de carótidas internas: reporte de caso. Anales de Radiología México, 2016;15(2):161-4.

17. SANDOE C, KINGSTON W. Exercise headache: a review. Current Neurology and Neuroscience Reports, 2018; 18(28).

18. TURNER J. Exercise-related headache. Curr Sports Med Rep, 2003;2(1): 15-17.

19. UPADHYAYA P, et al. Primary Exercise Headache. Current Neurology and Neuroscience Reports. 2020; 20(9).

20. ZIMMERMAN NB. Occlusive vascular disorder of the upper extremity. Hand Clin, 1993; 9(1): 139-50. 